\title{
Riemann-DTI Geodesic Tractography Revisited
}

\author{
Luc Florack, Rick Sengers, Stephan Meesters, Lars Smolders, \\ and Andrea Fuster
}

\begin{abstract}
Clinical tractography is a challenging problem in diffusion tensor imaging (DTI) due to persistent validation issues. Geodesic tractography, based on a shortest path principle, is conceptually appealing, but has not produced convincing results so far. A major weakness is its rigidity with respect to candidate tracts it is capable of producing given a pair of endpoints, showing a tendency to produce false positives (such as shortcuts) and false negatives (e.g. if a shortcut supplants the correct solution). We propose a new geodesic paradigm that appears to overcome these problems, making a step towards semi-automatic clinical use. To this end we couple the DTI tensor field to a family of Riemannian metrics, governed by control parameters. In practice these parameters may allow for edits by an expert through manual selection among multiple tract suggestions, or for bringing in a priori knowledge. In this paper, however, we consider an automatic, evidence-driven procedure to determine optimal controls and corresponding tentative tracts, and illustrate the role of edits to remediate erroneous defaults.
\end{abstract}

L. Florack $\cdot$ R. Sengers $(\bowtie) \cdot S$. Meesters $\cdot$ L. Smolders $\cdot$ A. Fuster Eindhoven University of Technology, Department of Mathematics \& Computer Science, NL-5600 Eindhoven, MB, The Netherlands

e-mail: H.J.C.E.Sengers@tue.nl

L. Florack

e-mail: L.J.M.Florack@tue.nl

S. Meesters

e-mail: S.P.L.Meesters@tue.nl

L. Smolders

e-mail: L.Smolders@ @student.tue.nl

A. Fuster

e-mail: A.Fuster@tue.nl

(C) The Author(s) 2021

E. Özarslan et al. (eds.), Anisotropy Across Fields and Scales,

Mathematics and Visualization, https://doi.org/10.1007/978-3-030-56215-1_11 


\section{Introduction}

Tractography aims at reconstructing bundles of nerve fibers in the brain (aka tracts) from diffusion weighted magnetic resonance imaging (DWI), the only neuroimaging technique enabling non-invasive in vivo imaging of the brain's fibrous structure. Unfortunately, persistent issues curb clinical progress [14, 53]. Decades after its inception [5, 7, 23, 30, 38, 47], lack of consensus and even skepsis as to its clinical feasibility prevail. This has sparked new incentives to specifically address current limitations in quantitative evaluation studies and international competitions [9, 12, 13, 37, 39, 51].

The geometric rationale for Diffusion Tensor Imaging (DTI), in its original form proposed by O'Donnell et al. [43] and, from a somewhat different perspective, by Lenglet et al. [31], and subsequently adapted by Fuster et al. [21], Hao et al. [24, 25], and several others, stipulates that one can 'geometrize away' local diffusivity patterns inside the brain. The idea is to incorporate anisotropic diffusivity of water in brain white matter, viewed as a porous medium with an orientational preference along axon bundles [57], into the intrinsic geometry of a suitably defined, curved manifold (akin to the geometrization of gravitational forces in general relativity theory). A similar approach is taken by Aumentado-Armstrong et al. [4], where a curved manifold is used to model conductivity of electrical signals in the heart muscles. In the case of DTI a Riemannian manifold presents itself, since its defining metric represents a positive definite quadratic form (or inner product) that can be formally mapped one-to-one onto the DTI tensor. Due to its modest performance, however, geodesic tractography has been largely abandoned in return for other approaches. A notable exception is the probabilistic approach by Hauberg et al. [26] and Schober et al. [55].

Our goal is to provide a versatile deterministic Riemann-DTI geometric paradigm for DTI geodesic tractography, revisiting original ideas (loc. cit.), which overcomes some of the main weaknesses, such as producing false positives (e.g. shortcuts) and false negatives (e.g. a shortcut is obscured by the correct solution). Since the mapping of DTI data to biologically meaningful tracts is generally ill-posed, we aim for a flexible metric equipped with control parameters. This admits adaptation to fiducial 'ground truth' tracts, in which data-extrinsic knowledge may be incorporated (e.g. via manual edits by an expert or via machine learning). The parameters control a locally smooth, spatially varying so-called '3-bein', or triad, detailed in Sect. 2 (cf. Savadjiev et al. [54] and Piuze et al. [48] for a similar idea in the context of myofiber geometry from DTI, based on Cartan's method of moving frames). In Sect. 3 we perform experiments to illustrate the theory, provide proof of principle, and present results in the context of a simple DTI-tractography phantom for the sake of illustration. There are no obstacles for application on more sophisticated simulated or real data. 


\section{Theory}

Differential geometric approaches in DWI are not new [1, 3, 15, 20-22, 24, 25, 31$33,43,46,48]$. The premise underlying the Riemann-DTI paradigm is that tissue microstructure imparts non-random barriers to water diffusion [8, 57]. The fibrous nature of brain white matter, comprising bundles of elongated axons connecting nerve cells in surrounding grey matter regions, facilitates mobility of water molecules along fiber directions. Since DTI captures the main diffusion anisotropy, it is natural to stipulate a Riemannian metric proportional to the inverse of the diffusion tensor. In this way, a relatively large mean free path is tantamount to a relatively short Riemannian distance, so that the problem of tractography can be related to a geodesic ('shortest path') problem. Candidate tracts can then be obtained by direct integration of the geodesic equations, by functional minimization of the Riemannian length (or related cost) functional for curves with fixed endpoints, or (with some care) by inference from the Hamilton-Jacobi equation [28, 45, 50, 52].

Despite the appealing heuristics supporting the geometric paradigm, there are serious caveats we need to take into consideration:

1. In a geodesically complete space any pair of points is connected by at least one geodesic, raising the issue of 'false positives' (curves not corresponding to meaningful tracts).

2. A well-posed relation between geodesics as 'paths of least resistance' and meaningful neural tracts is not self-evident. Tissue microstructure, which remains unresolved at scanner resolution, induces mesoscopic diffusivity patterns involving more complex factors than plain presence or absence of nerve axons [41, 42, 44]. As a consequence, there may be many a priori equally viable microstructural explanations for any given DTI image, ${ }^{1}$ so that a one-to-one mapping between DTI and Riemannian metric is unlikely to work for tractography.

We will address both concerns and outline our strategy towards an improved framework.

Ad caveat 1: Geodesic completeness, tantamount to (huge) redundancy, may be used to our advantage, provided two conditions are met:

(i) Meaningful tracts correspond, to acceptable approximation and at least piecewise, to geodesics.

(ii) One can identify true (or reject false) positives, based on some deterministic or probabilistic criterion.

The first condition is our main hypothesis, the second one is a constitutional part of the geodesic tractography problem. The problem then boils down to finding 'the right' metric together with effective connectivity criteria for pruning its geodesics $[2,50,56]$. Streamline tractography, in its simplest form a singular limit of geodesic

\footnotetext{
${ }^{1}$ This fact also implies that 'ground truth' simulations must be interpreted with great care in order not to penalise experimental results that deviate from the stipulated ground truth used to create a phantom if such results are equally compatible with data evidence.
} 
tractography based on a degenerate metric, lacks completeness, a direct consequence of its first order nature, which prohibits generic endpoint constraints. As a result, odds are that two fiducial endpoints (picked by an authoritative expert, say) fail to be connected, leaving us in a quandary how to repair for 'false negatives'. Clearly, geodesic completeness requires (at least) second order schemes.

Ad caveat 2: To allow for prior knowledge or retrospective corrections we do not determine a unique metric in terms of DTI data evidence a priori. Instead we aim for optimal control parameters for a family of metrics and induced geodesics in a joint (semi-)automated procedure. The premise is that, due to unknown microstructural factors, apparent diffusivities reflect fiber orientations at best qualitatively. By investigating the parameter unfolding of the family of metrics along with geodesic pruning we may investigate whether a stable result compatible with ground truth can be achieved (bearing in mind footnote 1). To this end we have conducted a feasibility study on the Fibercup [13, 49] simulator to clarify all conjectured features of our approach in a simplified context. There are no fundamental obstructions for application to more sophisticated phantoms or to real data, but this elaboration is left for future work.

For computational reasons one could employ any coordinate basis on the tangent bundle $T M$ induced by an arbitrary coordinate map. Such a basis is commonly denoted by $\left\{\partial_{i} \doteq \partial / \partial x^{i}\right\}_{i=1,2,3}$. (A natural choice would be to employ the same coordinates as those given by a Cartesian coordinate frame of the associated Euclidean space.) However, given a suitable metric $g_{i j}$, we may instead opt for a special, $g$-orthogonal (non-coordinate, or anholonomic) basis $\left\{e_{a}\right\}_{a=1,2,3}$. This is the triad alluded to in Sect. 1. If we write the new basis vectors in terms of linear combinations of the coordinate vectors, ${ }^{2}$

$$
e_{a}=e_{a}^{i} \partial_{i}
$$

then the coefficients $e_{a}^{i}$ define the transformation matrix relating the general coordinate basis to the triad. We may define a new metric holor with entries $h_{a b}$ relative to the triad by the standard change-of-basis formula:

$$
g_{i j} e_{a}^{i} e_{b}^{j}=h_{a b}
$$

The triad can be chosen so as to put $h_{a b}$ into a convenient form. In order to illustrate this 'gauge fixing', let us assume that the dual metric $g^{\text {inv }}$ is identified with the DTI matrix $D$, as originally proposed. Take $h=\operatorname{diag}\left(1 / \lambda^{1}, 1 / \lambda^{2}, 1 / \lambda^{3}\right)$, in which the $\lambda^{a}$ are the eigenvalues of $D$. In the parlance of classical matrix theory [27], $e_{a}$ is then the eigenvector of $D$ associated with $\lambda^{a}$, which, in turn, defines a rank-one matrix $Z_{a}=e_{a} \otimes e_{a}$ ('Frobenius covariant'). Equation (2) is the geometrical counterpart of the classical Lagrange-Sylvester matrix decomposition, $D=\sum_{a=1}^{3} \lambda^{a} Z_{a}$, and the eigenvalues are our controls. We may vary the $\lambda^{a}$ and observe the effect on the

\footnotetext{
${ }^{2}$ The Einstein summation convention for identical upper/lower index pairs applies throughout in tensorial equations.
} 
metric, induced geodesics, and ultimately fiber tracts. This parameter freedom unifies originally proposed models for the connection between DTI and Riemannian metric [21, 24, 25, 31, 43], since all instances can be obtained by slick (local or global) choice of $\lambda^{a}$. This includes streamline tractography via singular perturbation theory.

To find a geodesic we fix seed and target points, $A$ and $B$ say, and minimize the length functional for curves connecting these points. This can be extended to any pair of regions provided one employs an efficient algorithm. Solving the Hamilton-Jacobi equation (with the help of fast marching methods) seems attractive in this respect, but, by design, provides only global minimizers, and is therefore not likely to solve the shortcut problem. Instead we opt for direct, coarse-to-fine minimization of the parametrization invariant functional

$$
L_{g}(\gamma)=\int_{t_{A}}^{t_{B}} \sqrt{g_{i j}(x(t)) \dot{x}^{i}(t) \dot{x}^{j}(t)} d t,
$$

in which

$$
\gamma:\left[t_{A}, t_{B}\right] \rightarrow \mathbb{R}^{3}: t \mapsto x(t)
$$

is an arbitrarily parametrized curve connecting $A=x\left(t_{A}\right)$ and $B=x\left(t_{B}\right)$ in $\mathbb{R}^{3}$ and $\dot{x}$ denotes its derivative. We embed the metric in a multiresolution family, with scale parameter $\sigma \in \mathbb{R}^{+}$, according to the multiplicative scheme proposed by Florack et al. for positive symmetric matrices $[16,18]$, viz. if $g$ is the Gram matrix with entries $g_{i j}$, then

$$
g(x, \sigma)=\exp \left(\left(\phi_{\sigma} * \ln g\right)(x)\right)
$$

in which exp and $\ln$ are matrix-exp and matrix-log, $\phi_{\sigma}$ is the $L^{1}$-normalised isotropic Gaussian kernel of scale $\sigma[17,19,29,34]$, and $*$ denotes entry-wise convolution. (This definition ensures that the dual of a blurred metric equals the blurred dual metric.) Since the metric can be shown to become Euclidean in the $\sigma \rightarrow \infty$ limit under suitable, weak conditions, the asymptotic minimizer is the straight line connecting $A$ and $B$. As one gradually decreases $\sigma$, this minimizer (geodesic at resolution $1 / \sigma$ ) is expected to deform likewise gradually, while adapting to the refined metric (at this level of rigor we ignore the problem of scale space bifurcations, cf. Damon [10, 11]). A higher resolution geodesic can then be found by adding a few control points roughly equidistantly along the curve in proportion to resolution, and minimizing the multivariate function of the control points representing our discretization of Eq. (3). Now suppose we have a (local) minimizer at some scale $\sigma$, then along with a scale refinement $\sigma \rightarrow \sigma-d \sigma$ (we take $d \sigma \propto \sigma$ ) we increment the number of control points along the curve, and seek a new minimizer in the vicinity of the previous one. In this way one can arbitrarily refine the geodesic curve (until grid scale, if needed), cf. Fig. 1.

The multiresolution scheme for geodesics sketched above applies to each fixed member of the $\left(\lambda^{1}, \lambda^{2}, \lambda^{3}\right)$-family of metrics, and should not be confused with optimization with respect to the unfolding of this family as a function of $\left(\lambda^{1}, \lambda^{2}, \lambda^{3}\right)$. The details of this are given in the next section, notably Eq. (6). 


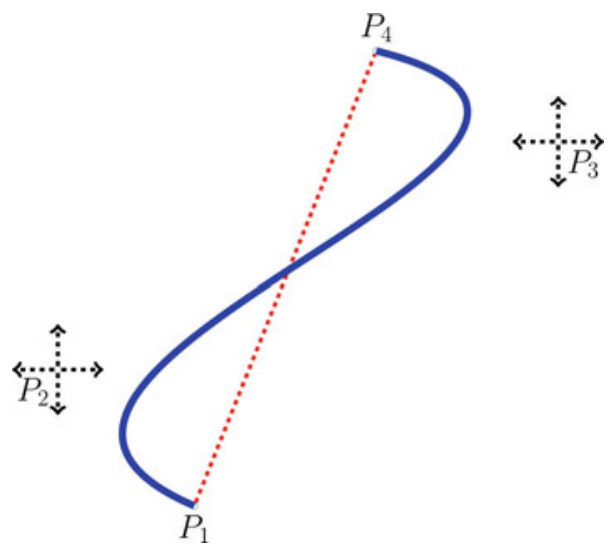

Fig. 1 Coarse-to-fine minimization of the length functional in Eq. (3) is implemented as an iterative multivariate function minimization in which the number of variables gradually increases in proportion to resolution. The control points represent a Bezier curve (blue) which by default we initialize by the unique Euclidean geodesic (dotted line), although this initialising curve may be manually overruled. In this example there are two control points, $P_{2}$ and $P_{3}$ (recall that the endpoints, $P_{1}$ and $P_{4}$ in this case, remain fixed). Note that the control points in general do not lie on the curve itself. These points are part of the 'behind-the-scenes' machinery in the minimization procedure and in themselves do not have any anatomical meaning. However, given $k$ points in space, we may construct a Bezier curve through these points (although not uniquely). This may be useful for manual initialisation or correction by an expert, based on anatomical landmarks.

\section{Experiments}

In the following experiments we use an operational procedure to automatically select 'the right' (anisotropic) scaling of the control parameters $\lambda^{a}$ for the triad, relative to the original eigenvalues of the diffusion tensor. To begin with, we have opted for a single, global parameter $\varepsilon \in(0,1]$, introduced so as to rescale the diagonal metric $h^{\text {inv }}$ (the dual of $h$ in Eq. (2)) by ${ }^{3} \operatorname{diag}(1, \varepsilon, \varepsilon)$. The global character of $\varepsilon$ allows us to leave $\lambda^{1}$ unscaled, since any scaling of $\lambda^{1}$ can be absorbed into $\varepsilon$. Effectively this yields an $\varepsilon$-parametrized family of Riemannian metrics $g_{\varepsilon}$, replacing the unscaled metric $g=g_{\varepsilon=1}$ in Eqs. (2), (3) and (5). However, we only use this scaling whenever the fractional anisotropy (FA) [6] of the diffusion tensor is large enough (here we choose $\mathrm{FA}=0.15$ as the ad hoc threshold) to ensure a well-defined main eigendirection. ${ }^{4}$ Below this threshold the diffusion tensor is kept unscaled. As a result we obtain an $\varepsilon$-parametrized family of geodesics $\gamma_{\varepsilon}$, cf. Eq. (4). These geodesics are computed one

\footnotetext{
${ }^{3}$ In principle, the control parameter triple $\left(\lambda^{1}, \lambda^{2}, \lambda^{3}\right)$ permits local adaptation leading to a 3parameter family in every voxel. The singular limit $\varepsilon=0$ corresponds to streamline tractography and is excluded for its geodesic incompleteness. Values $\varepsilon>1$ may affect the ordering of the eigenvalues of $h^{\text {inv }}$.

${ }^{4}$ This ad hoc anisotropy threshold calls for a more rigorously motivated alternative, but it serves our purpose in this feasibility study, viz. to ensure well-posedness.
} 
by one by the coarse-to-fine scheme outlined at the end of Sect. 2 for each fiducial pair of endpoints and each setting of $\varepsilon$.

Subsequently each geodesic thus obtained is quantitatively evaluated in terms of the tract length unbiased, nonlinear connectivity functional $[2,50,56]$

$$
C\left(\gamma_{\varepsilon}\right)=\frac{L_{\eta}\left(\gamma_{\varepsilon}\right)}{L_{g}\left(\gamma_{\varepsilon}\right)},
$$

in which $\eta$ denotes the standard Euclidean metric (so that $L_{\eta}(\gamma)$ is the usual length of $\gamma)$, recall Eq. (3). Note that in the denominator we use the unscaled diffusion metric $g=g_{\varepsilon=1}$, for which the curve $\gamma_{\varepsilon}$ is, in general, not a geodesic (unless $\varepsilon=1$ ). Using the scaled length $g_{\varepsilon}$ in the denominator would entail a bias towards $\varepsilon=1$. Each geodesic $\gamma_{\varepsilon}$ will almost surely not correspond to a streamline (unless both endpoints are on the streamline), which implies that there exists a $t \in(0,1)$ such that the tangent vector $\dot{\gamma}_{\varepsilon}(t)$ has a component in the direction of a non-principal eigenvector of the diffusion tensor. Since this component scales with $\varepsilon^{-1}$, the connectivity would vanish for $\varepsilon \rightarrow 0$ even if the geodesic remains (approximately) the same curve. Instead, the $\varepsilon$ control parameter merely serves to single out an optimal, metric-compatible geodesic through a suitable anisotropic scaling of the metric tensor. The connectivity criterion for the latter is based on data evidence, viz. average apparent diffusivity, which should not involve $\varepsilon$. Connectivity $C\left(\gamma_{\varepsilon}\right)$ is thus some average measure of diffusivity along $\gamma_{\varepsilon}$. Parameter values

$$
\varepsilon^{*} \in \underset{\varepsilon \in[\delta, 1]}{\arg \max } C\left(\gamma_{\varepsilon}\right),
$$

for some fixed $0<\delta \ll 1$, at which locally ${ }^{5}$ optimal connectivity is attained, are tractspecific sharpening parameters, affected in a still unknown manner, by underlying microstructure. The corresponding tracts $\gamma_{\varepsilon^{*}}$ are called optimal tracts.

As illustrated in Fig. 2 for the Fiberfox-reconstructed Fibercup phantom with added Riccian noise [40] (cf. Fillard et al. for a detailed description [13] of the original Fibercup) only optimal tracts are retained. Since it is known that the inverse metric has certain shortcomings (e.g. see [24]), we also employed our automated procedure in combination with one of the other metric proposals in the literature, namely the adjugate metric $[21,22]$. We chose this one, because it is derived from first principles of the underlying diffusion process and in addition it's very easy to implement. In Fig. 2, the optimal tracts found for the inverse (bottom-left) and adjugate DTI metric (bottom-right) emerge for different optimal parameter values, but are, remarkably, virtually indistinguishable. As the adjugate DTI metric is essentially a local isotropic scaling of the inverse DTI metric, it is a noteworthy feature that we are able to obtain essentially the same tracts in both cases with a family of metrics characterised by a single, global parameter. After all, the governing geodesic equations for the inverse and adjugate are not the same. In practice, a broad range of parameter values (the high plateaux in the graphs) can be used to single out essentially the same tracts, making this procedure robust with respect to the precise choice of $\varepsilon$. The experiment shows that, in all cases and by virtue of proper placement of endpoints, correct tracts

\footnotetext{
${ }^{5}$ We define $\operatorname{argmax}_{x} f(x) \doteq\{x \mid f(y) \leq f(x)$ for all $y$ in a neighbourhood of $x\}$.
} 


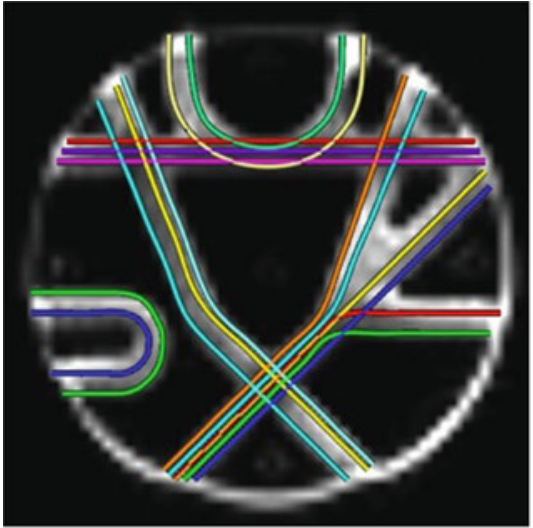

$\mathrm{C}\left(y_{\epsilon}\right)$

3.0

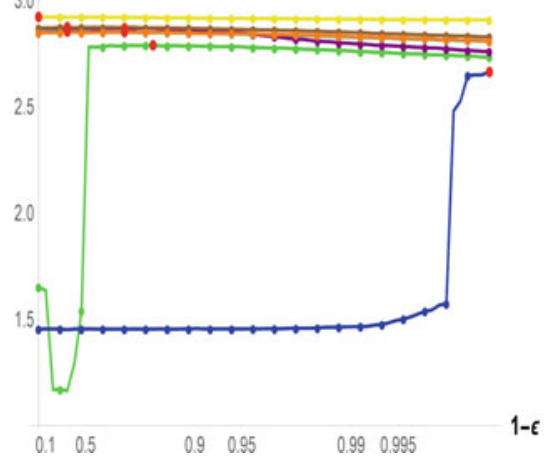

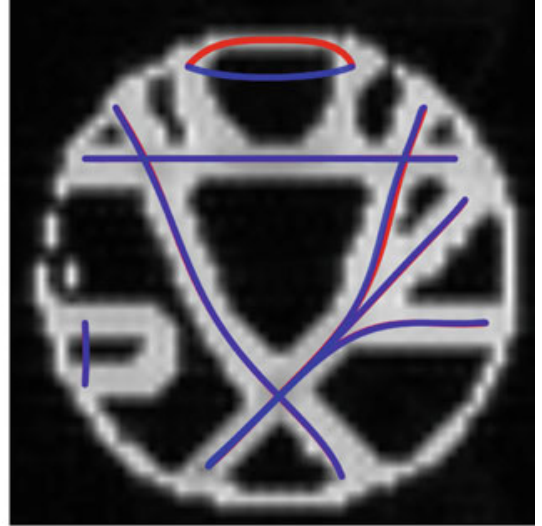

$\mathrm{C}\left(y_{\epsilon}\right)$

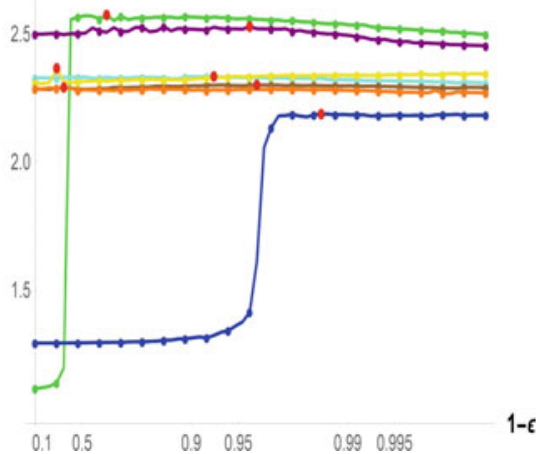

Fig. 2 (Top-left) Ground truth tracts (for various seed points); illustration adapted from Fillard et al. [13]. (top-right) Tracts obtained by using the unscaled inverse (red), respectively adjugate (blue) DTI tensor as the default metric for geodesic tractography. Both metrics produce shortcuts (false positives) instead of following the U-shape fibers, leaving false negatives. (middle-left) Pertract connectivity for the $\varepsilon$-parameter unfolding of the inverse DTI metric, $g=D^{\text {inv }}$. (middle-right) Idem for the adjugate DTI metric, $g=\operatorname{det} D D^{\text {inv }}$. Colors correspond to those used for the tracts in the bottom two figures. The red dots indicate the tract-specific parameter values $\varepsilon^{*} \in(0,1]$ for the metrics used for each optimal tract. This optimal parameter is not affected by a suitable (global) normalization of $g$, meaning that the absolute value of the connectivity is inherently meaningless. It is, however, the relative value that determines the optimal parameter. Note the logarithmic scale for $\varepsilon$. (bottom-left) Optimal results obtained by automatic optimization for inverse DTI metric ansatz. (bottom-right) Optimal results obtained by automatic optimization for adjugate DTI metric ansatz. The latter two results are virtually indistinguishable, but optimality is obtained at different control parameter settings. 

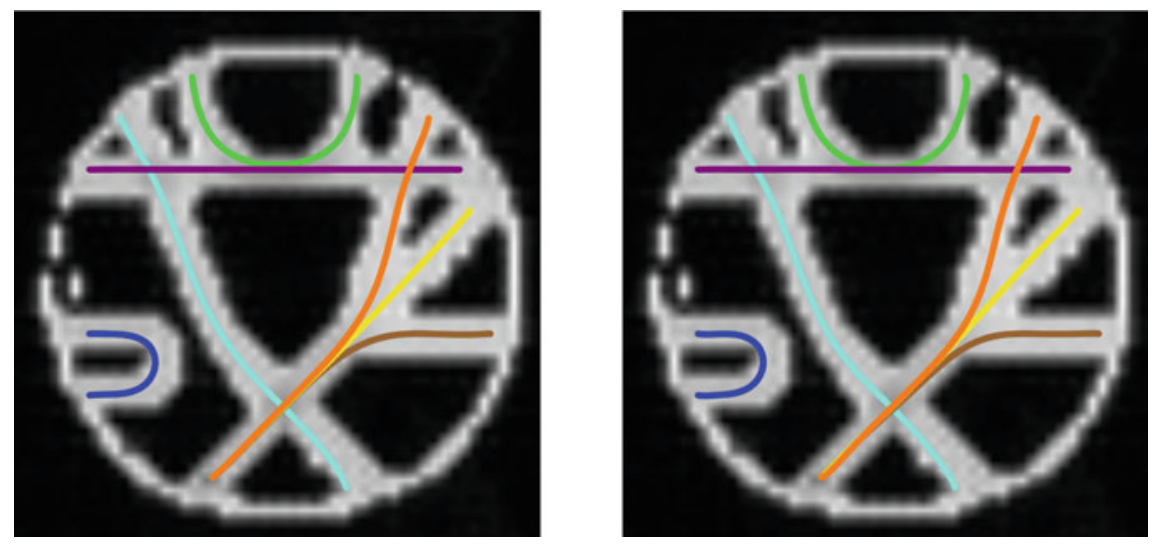

Fig. 2 (continued)

are found automatically, and, in particular, that shortcuts do not occur. To assess the robustness to placement of endpoints, in Fig. 3 the optimal tracts are computed for pairs of perturbed endpoints, demonstrated for the adjugate DTI metric.

Low connectivity typically corresponds to shortcuts. Figure 4 shows optimal tracts for each pair of corresponding (ground truth) seed and target points (top), as well as connectivities for optimal tracts between a selection of seed point and all remaining target points (bottom). For each pair of possible seed/target points, the optimal tract was determined by Eq. (7). Figure 4 shows two representative cases: one in which the stipulated ground truth yields the highest connectivity and a second one in which it is among the top but is still dominated by another tract.

Multiple local maxima of $C\left(\gamma_{\varepsilon}\right)$ generally exist, occurring at $\varepsilon \in\left\{\varepsilon_{1}^{*}, \ldots, \varepsilon_{k}^{*}\right\}$ say, corresponding to $k$ plausible tracts (recall Eq. (7) and footnote 5) that invariably include the stipulated 'ground truth', although the latter does not necessarily correspond to the global maximum, recall Fig. 4b. Figure 5 illustrates this case by another simulation (based on Fiberfox [40]), emblematic of an ambiguous configuration of kissing or crossing fiber tracts . Both interpretations are indeed confirmed, in the sense of corresponding to local optima of $C\left(\gamma_{\varepsilon}\right)$. Our current coarse-to-fine implementation, however, cannot handle scale space bifurcations of geodesics automatically, and, due to our default initialization by the Euclidean geodesic, automatically zooms in on the kissing tract. The crossing tract requires a (simple) manual edit to overrule the default initialization and encourage convergence to another solution. This edit and its effect is shown in Fig. 5, and further illustrated in Fig. 6. Here the default initialization by the Euclidean geodesic has been replaced by a quadratic Bezier curve forced to pass through a manually selected inclusive ROI called an AND gate (green circle), while connectivity maximization for this control point has been constrained to this neighbourhood. Note that, for the inverse as well as the adjugate diffusion tensor ansatz, Fig. 6, boundary extrema $\gamma_{\varepsilon}$ are found near $\varepsilon=1$, illustrating the same inclination to produce shortcuts as $\gamma_{1}$ for the unscaled metric. Closer to the optimal $\varepsilon^{*} \in \operatorname{argmax}_{\varepsilon \in[\delta, 1]} C\left(\gamma_{\varepsilon}\right)$ we observe correct convergence, though. 


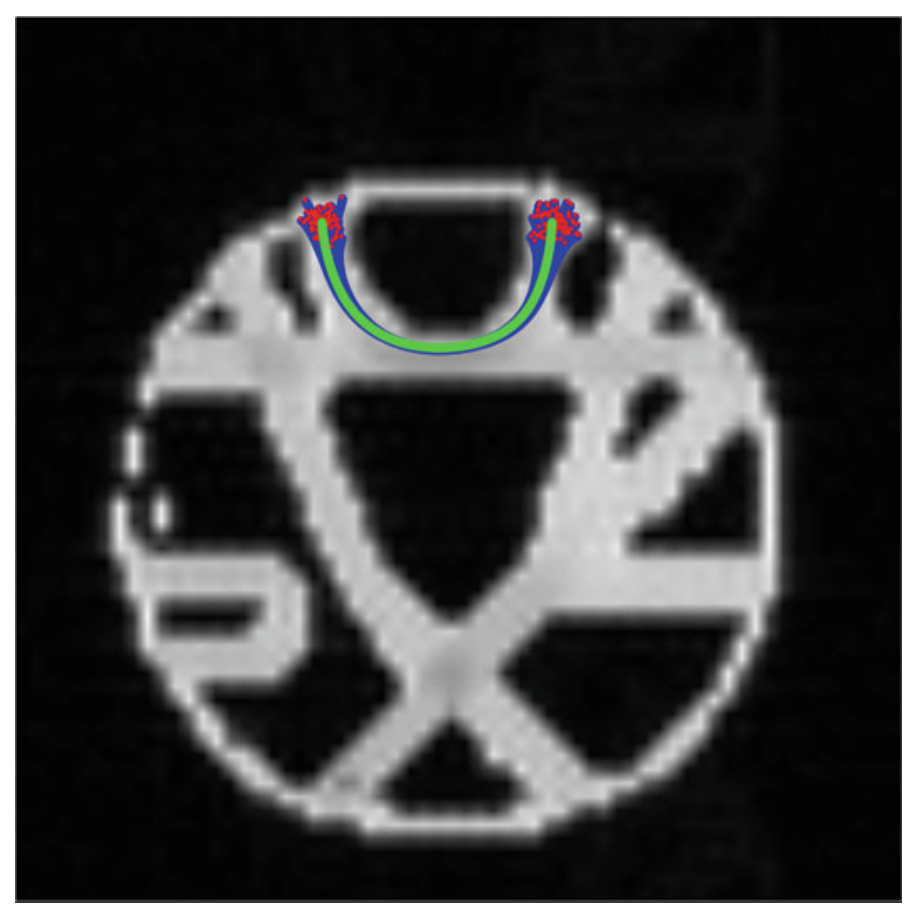

Fig. 3 For each endpoint of the optimal green curve (cf. Fig. 2) the red points are generated from a multivariate normal distribution centered around the endpoints with covariance matrix $\Sigma=$ $\operatorname{diag}(0.5,0.5)$. The blue tracts are the optimal tracts for pairs of such perturbed endpoints by using the adjugate DTI metric ansatz. The green curve has a connectivity value of 2.749 and the mean connectivity of all perturbed tracts is 2.708 with a standard deviation of 0.037 . Visually, the green curve is a good representative of this bundle of tracts as a whole and its connectivity is approximately one standard deviation away from the mean.

In Fig. 7, the left Cortical Spinal Tract of the ISMRM 2015 Tractography challenge data [35] is used to illustrate the operational procedure on a 3D dataset. Quantitative measures used in the challenge are the overlap (OL), overreach (OR) and $F_{1}$ scores, see Côté et al. [9]. OL is defined as the number of voxels through which both the reconstruction and the ground truth pass, normalized by the number of ground truth voxels. OR is the number of voxels through which the reconstruction passes but the ground truth does not, normalized by the number of reconstructed voxels. These two quantities can be interpreted as the true positive rate and the false discovery rate. The $F_{1}$ score is the harmonic mean of OL and $1-$ OR. Our tractography results in $\mathrm{OR}=0.324$, OL $=0.375$, leading to $F_{1}=0.427$, ranking it in the top $40 \%$ of the submitted challenge results based solely on $F_{1}$ scores. It is particularly noteworthy that this DTI-based procedure has a performance comparable to HARDI type methods, outperforming other DTI-based methods applied to the CST [36]. 

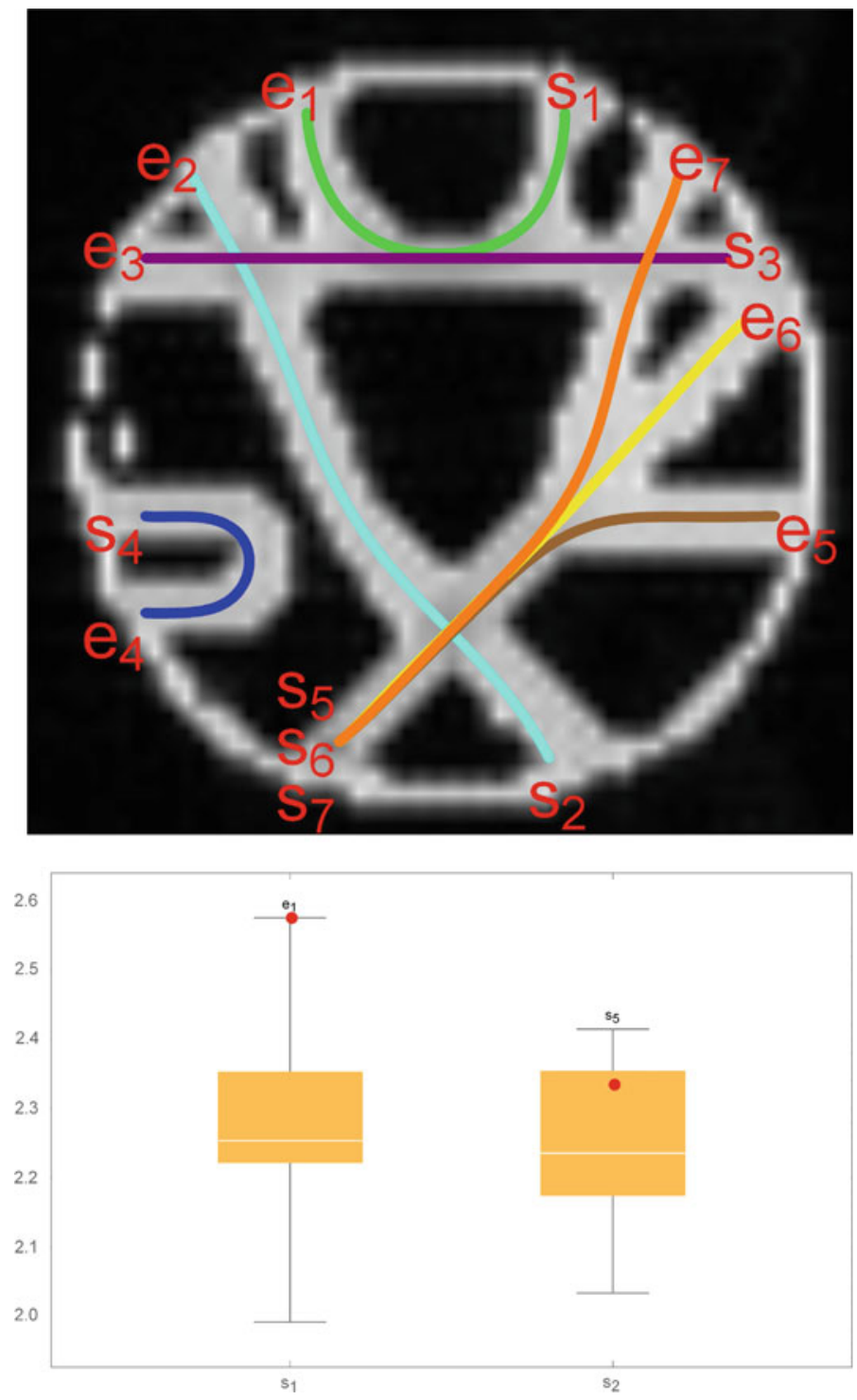

Fig. 4 (Top) Seed and target point labeling: $s_{i}=$ start of tract, $e_{i}=$ end of tract; the attached number $i \in\{1, \ldots, 7\}$, refers to the tract label. (bottom) Box-and-whisker plot of connectivity in the adjugate DTI metric versus seed point. For the seed points $\mathrm{s}_{1}$ and $\mathrm{s}_{2}$ the boxplot above it shows the optimal connectivity of that seed point to every other target point $\mathrm{s}_{j}$ and $\mathrm{e}_{j}$. Endpoints that yield the highest connectivity are indicated on top on the boxplot. The red dots indicate the connectivity value for the stipulated ground truth, i.e. the tract running from $\mathrm{s}_{i}$ to $\mathrm{e}_{i}$, for each tract label. 

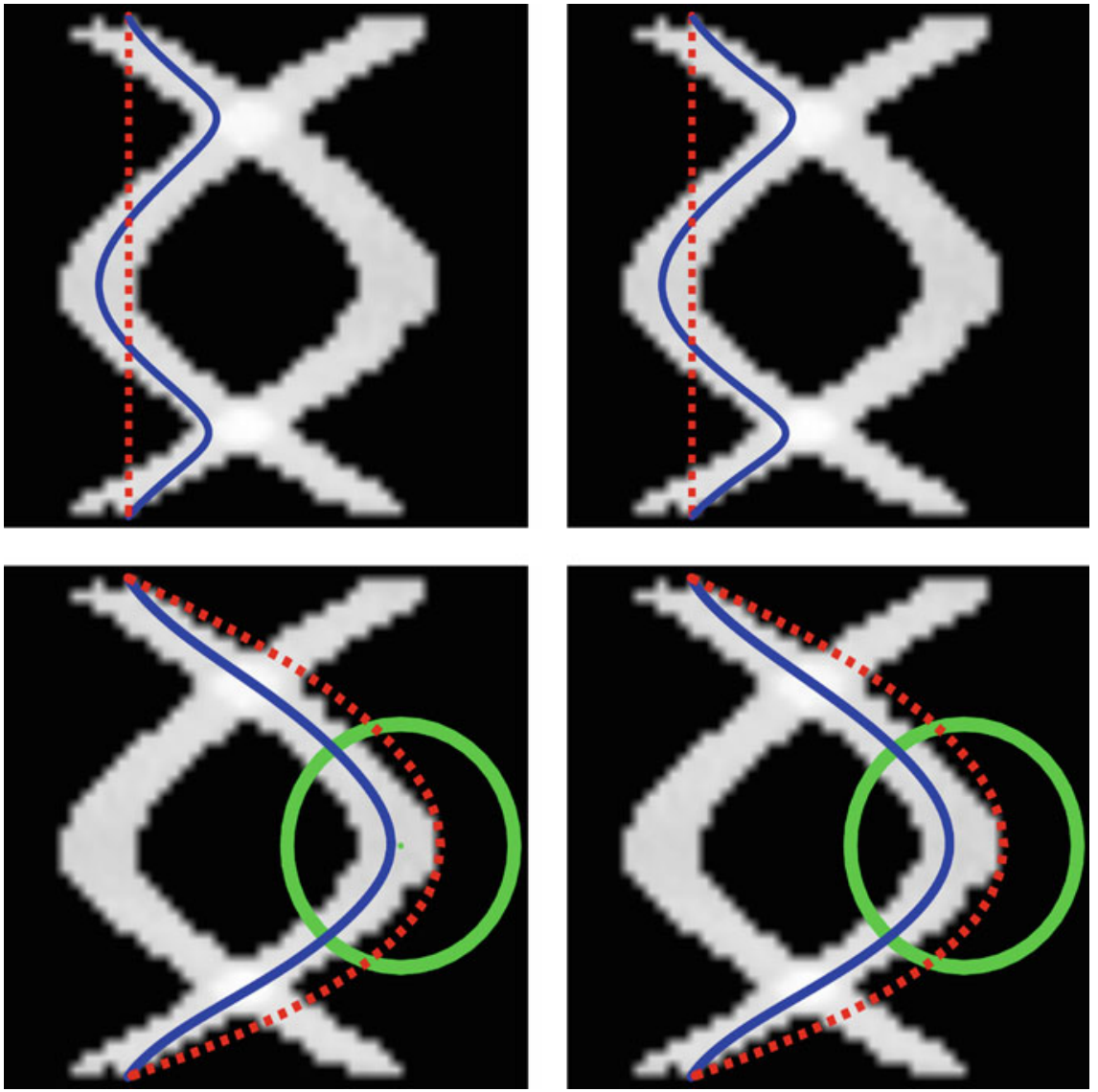

Fig. 5 Simulation with kissing/crossing fibers. Seed and target points are invariably taken to lie in upper and lower left corner. Note that there are two plausible solutions. The left two figures pertain to the (connectivity pruned) $\varepsilon$-family induced by the inverse diffusion metric, the right two figures to that of the adjugate diffusion metric. In either case, our automatic procedure only finds one solution (blue curves). (top) The equally viable crossing scenario is due to the coarse-to-fine scheme with default initialization (red dotted Euclidean geodesic in the upper two figures). (bottom) By employing a restricted neighbourhood search region (AND gate, green circle) with suitable initialization (red dotted quadratic Bezier curve), the algorithm converges to the blue crossing geodesics in the bottom two figures, resolving the false negative result. 

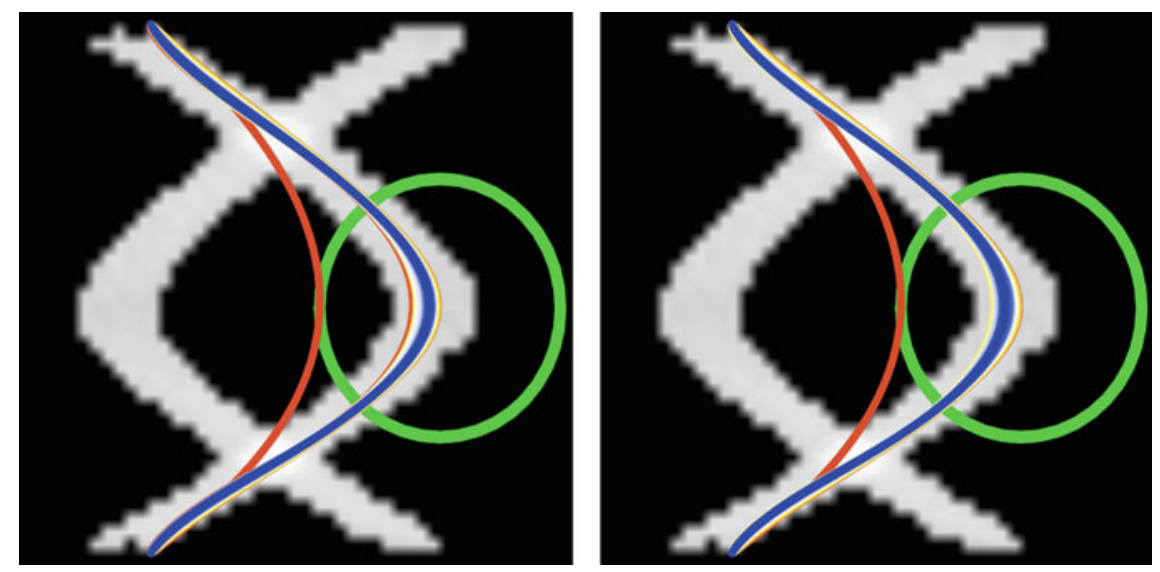

Fig. 6 Geodesics found by unfolding of the Riemannian metric family $g_{\varepsilon}$, Eq. (7) in combination with connectivity optimization, Eq. (6) for inverse (left) and adjugate (right) diffusion tensor ansatz $g=g_{1}$, the AND gate of Fig. 5 (green circle). Initialization is given by the quadratic Bezier curve in Fig. 5. Red-to-blue rainbow color coding shows the connectivity evolution as a function of $\varepsilon$, with red indicating low connectivity tracts and blue indicating the (unique) connectivity optimized result for $\varepsilon^{*}=\operatorname{argmax}_{\varepsilon \in[\delta, 1]} C\left(\gamma_{\varepsilon}\right)$.

\section{Conclusion and Discussion}

We have proposed a modified Riemann-DTI geodesic tractography framework. The modification entails the embedding of a DTI induced metric into a family of metrics designed for the purpose of tractography, furnished with an operational scheme for tract-specific optimization of metric and geodesic(s) for any given pair of endpoints based on a nonlinear connectivity functional.

In a simple experiment based on the Fibercup simulator we conclude that all local connectivity maxima correspond to plausible tracts that consistently follow high diffusivity pathways and avoid, as much as logically possible, inconsistent regions. For instance, despite a rather simplistic connectivity measure, all ground truth tracts are reconstructed provided seed and target points are (roughly) correctly placed, i.e. in such a way that a plausible fiber does indeed exist, recall Fig. 2. The robustness of our procedure with respect to the placement of endpoints is demonstrated in Fig. 3, where tracts reconstructed using slightly perturbed seed and target locations, remained in close proximity to the original one.

It is essential, in this respect, to appreciate the ill-posed nature of tractography. Although the generation of diffusivity patterns from a given tract configuration is robust (albeit model-dependent), this process is not invertible. The equivalence class of tract configurations consistent with a given diffusivity pattern may contain many 'metamers', so to speak. The actual power of the proposed method is precisely its ability to generate multiple representatives from this equivalence class, so that no a priori bias is introduced. In clinical practice this could help an expert to reject 

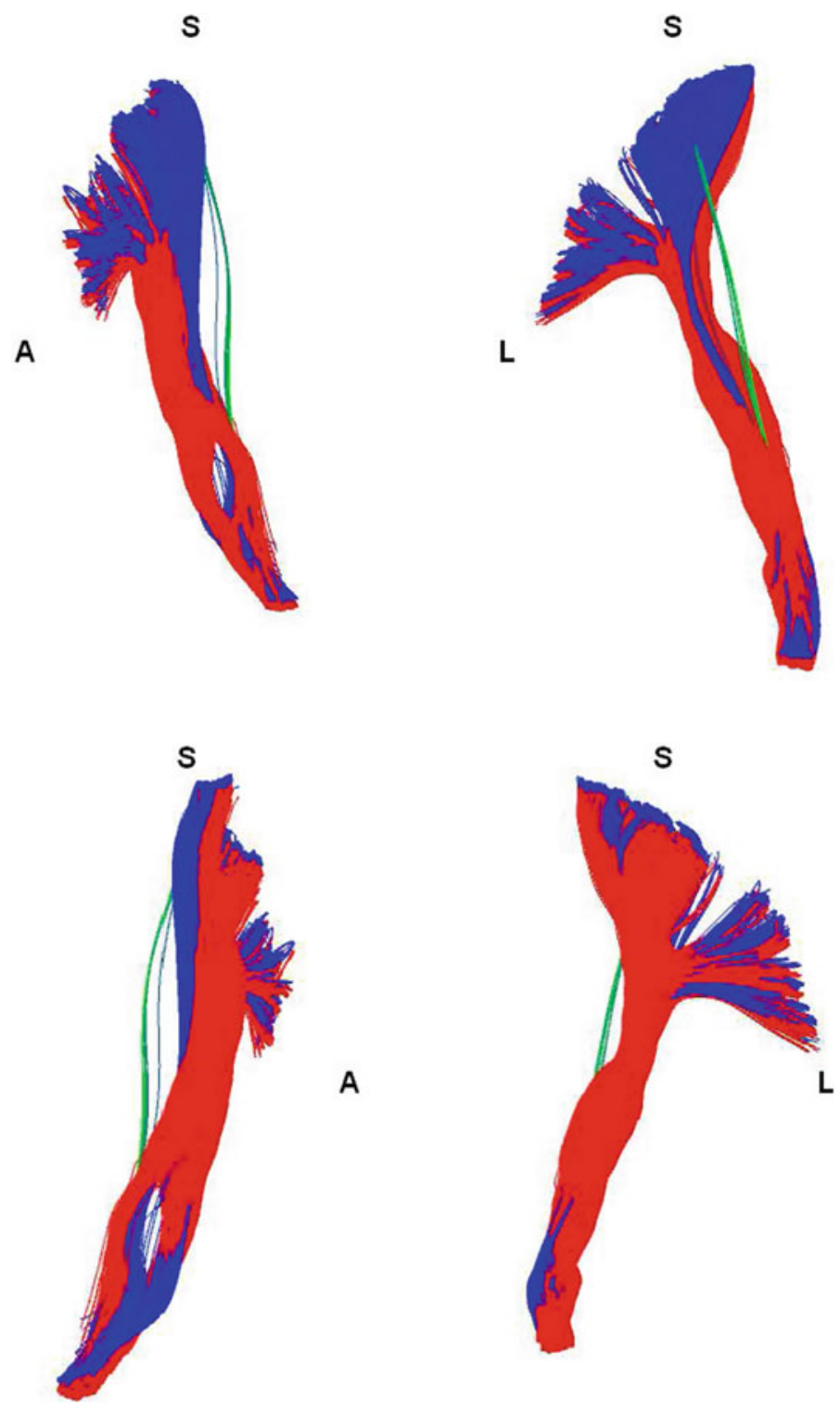

A

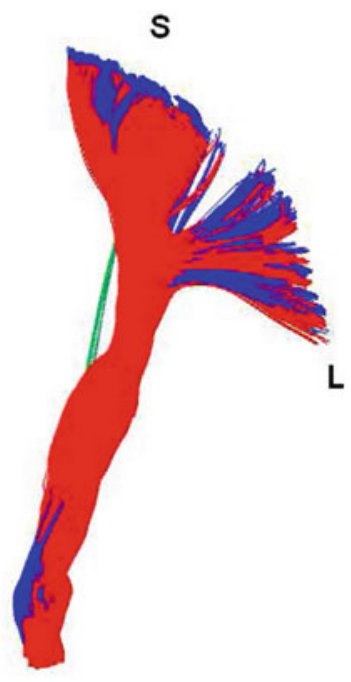

Fig. 7 Comparison of the Left Cortical Spinal Tract (CST) between the ground truth (red) and connectivity-optimal geodesics according to Eqs. (6) and (7) in which the base metric is the adjugate DTI-metric. The geodesics are color-coded according to their connectivity value, which increases from green to blue. The depicted views are labeled with S(uperior), A(nterior) and L(eft) to determine the plane and orientation of the cross-section. On visual inspection of the multiple views of the CST, the optimal geodesics lie close to the ground truth. Using more refined connectivity measures or a criterion to remove single tracts, the results can be improved. This can relieve us from those spurious tracts that are seemingly leaving the bundle and clearly have a lower connectivity than the rest of the tracts. A selection of 3000 ground truth tracts were extracted from the CST to determine seed and target points for the geodesics. 
false positives (if not eliminated automatically), based on data-extrinsic anatomical insight, in favour of selecting one of the remaining plausible candidate tracts, i.e. without being confronted with false negatives. Ideally such candidates present themselves, viz. as one of the local optima actually found by our automatic scheme.

False negatives may nevertheless occur if local maxima are missed due to inadequate numerical optimization. We have indicated how this problem may be mitigated through a simple manual edit by an expert. The delineation of an AND gate through which one believes tentative tracts of interest should pass, or the sketch of a coarse initialization curve between the endpoints, overruling the default straight line segment, both make it more likely to drive numerical optimization to the correct local maximum.

To prevent scaling of the diffusion tensors in a non-sensical manner, their eigenvalues are only affected whenever an FA threshold was exceeded in order to ensure a well-defined main eigendirection of the diffusion tensor. This treshold was based on experiments with the Fiberfox-reconstructed Fibercup phantom, but needs to be adjusted for tractography on real data. This ad hoc approach should be considered a first stepping stone towards a more refined technique combining the scaling of eigenvalues with the anisotropy of the tensor and the noise level of the data. Highly anisotropic tensors have a relatively large first eigenvalue and may tolerate a more severe scaling, since they provide more evidence for an articulated orientation. Since many low FA regions contain complex fiber structures, not scaling the DTI tensors there may influence the shape of the geodesics. In these areas we do not expect to get accurate results, since DTI is not able to resolve complex fiber structures adequately. However, scaling of the tensors enhances any noise present in the data and may increase the number of false positives, especially when the signal-to-noise-ratio is very low.

We stress that 'most likely' fiber candidates are obtained for any pair of endpoints in terms of local optimality of connectivity. Recalling Fig. 4, note that even though tract $s_{2}-e_{2}$ has a high connectivity value, maximal connectivity is attained by the tract $\mathrm{s}_{2}-\mathrm{s}_{5}$. This effect can be ascribed to the construction of the family of metrics $g_{\varepsilon}$ as well as the conservative nature of the connectivity measure in Eq. (6). At the intersection of two bundles the diffusion tensors are (nearly) isotropic, leading to similar diffusivities in all directions. This isotropy $(\mathrm{FA} \leq 0.15)$ prevents anisotropic rescaling of the metric, as explained. Moreover, as the connectivity measure only accounts for average diffusion along a tract and does not penalize curvature, a bend in the tract does not decrease its connectivity, as the latter is based on diffusivity, not geometry. These effects combined result in a connectivity-optimal tract which differs from the ground truth one. This needs to be remedied by using more sophisticated connectivity measures, which will be application dependent and may require prior knowledge about the bundles of interest. Our generic connectivity measure has been motivated mainly by our desire to preserve true positives, not as a criterion to remove false positives, allowing a modular approach. Additional criteria for pruning false positives connecting a fiducial seed point to a volumetric target region (possibly the whole brain) will be studied in future work, including appropriate extensions of the proposed coarse-to-fine tractography algorithm. 
In the ISMRM Tractography challenge reconstructed tracts are interpreted as centerlines of a fiber with a thickness/resolution of $1 \mathrm{~mm}$ isotropic voxel. The OL, $\mathrm{OR}$ and $F_{1}$ scores were calculated based on such $1 \mathrm{~mm}$ voxels [36]. The tractography, however, was performed on the diffusion weighted images consisting of $2 \mathrm{~mm}$ isotropic voxels. This way we can only resolve detail up to the grid scale of $2 \mathrm{~mm}$ and quantification measures should take this resolution difference into account when using the affine transformation to convert between the two different spaces. By downscaling this minimal resolution of $1 \mathrm{~mm}$ to $2 \mathrm{~mm}$ we increase the $F_{1}$ score to 0.526 , ranking in the top $20 \%$ (as the only DTI based algorithm).

In this experiment details could be resolved up to grid scale, but if one would consider the effects of noise (e.g. in the acquisition process), this may lead to a different effective minimal resolution, determined by grid scale and noise level. The influence of different kinds of noise (e.g. acquisition noise, seeding errors) needs to be addressed in future studies in which the control-triad framework is combined with uncertainty quantification.

These experiments confirm our main conjecture that the potential of DTI has not yet been fully exploited in tractography.

Acknowledgments This work is part of the research programme 'Diffusion MRI Tractography with Uncertainty Propagation for the Neurosurgical Workflow' with project number 16338, (partly) financed by the Netherlands Organisation for Scientific Research (NWO).

The work of Andrea Fuster is part of the research programme of the Foundation for Fundamental Research on Matter (FOM), which is financially supported by the Netherlands Organisation for Scientific Research (NWO).

\section{References}

1. Arsigny, V., Fillard, P., Pennec, X., Ayache, N.: Log-Euclidean metrics for fast and simple calculus on diffusion tensors. Magn. Reson. Med. 56(2), 411-421 (2006)

2. Astola, L., Florack, L., ter Haar Romeny, B.: Measures for pathway analysis in brain white matter using diffusion tensor images. In: Karssemeijer, N., Lelieveldt, B. (eds.) Proceedings of the Twentieth International Conference on Information Processing in Medical ImagingIPMI 2007 (Kerkrade, The Netherlands), Lecture Notes in Computer Science, vol. 4584, pp. 642-649. Springer, Berlin (2007)

3. Astola, L., Fuster, A., Florack, L.: A Riemannian scalar measure for diffusion tensor images. Pattern Recognit. 44(9), 1885-1891 (2011)

4. Aumentado-Armstrong, T., Kadivar, A., Savadjiev, P., Zucker, S.W., Siddiqi, K.: Conduction in the heart wall: helicoidal fibers minimize diffusion bias. Sci. Rep. 8(1), 7165 (2018). https:// doi.org/10.1038/s41598-018-25334-7

5. Basser, P.J., Pajevic, S., Pierpaoli, C., Duda, J., Aldroubi, A.: In vivo fiber tractography using DT-MRI data. Magn. Reson. Med. 44(4), 625-632 (2000)

6. Basser, P.J., Pierpaoli, C.: Microstructural and physiological features of tissues elucidated by quantitative-diffusion-tensor MRI. J. Magn. Reson. Ser. B 111(3), 209-219 (1996)

7. Batchelor, P.G., Moakher, M., Atkinson, D., Calamante, F., Connelly, A.: A rigorous framework for diffusion tensor calculus. Magn. Reson. Med. 53, 221-225 (2005)

8. Beaulieu, C.: The basis of anisotropic water diffusion in the nervous system-a technical review. NMR Biomed. 15(7-8), 435-455 (2002) 
9. Côté, M.A., Girard, G., Boré, A., Garyfallidis, E., Houde, J.C., Descoteaux, M.: Tractometer: towards validation of tractography pipelines. Med. Image Anal. 17, 844-857 (2013)

10. Damon, J.: Local Morse theory for solutions to the heat equation and Gaussian blurring. J. Differ. Equ. 115(2), 368-401 (1995)

11. Damon, J.: Generic properties of solutions to partial differential equations. Arch. Ration. Mech. Anal. 353-403 (1997)

12. De Santis, S., Drakesmith, M., Bells, S., Assaf, Y., Jones, D.K.: Why diffusion tensor MRI does well only some of the time: variance and covariance of white matter tissue microstructure attributes in the living human brain. Neuroimage 89, 35-44 (2014)

13. Fillard, P., Descoteaux, M., Goh, A., Gouttard, S., Jeurissen, B., Malcolm, J., RamirezManzanares, A., Reisert, M., Sakaie, K., Tensaouti, F., Yo, T., Mangin, J.F., Poupon, C.: Quantitative evaluation of 10 tractography algorithms on a realistic diffusion MR phantom. Neuroimage 56(1), 220-234 (2011)

14. Filler, A.: MR neurography and diffusion tensor imaging: Origins, history, and clinical impact of the first 50000 cases with an assessment of efficacy and utility in a prospective 5000-patient study group. Neurosurgery 65(4), 29-43 (2009)

15. Fletcher, P.T., Joshi, S.: Riemannian geometry for the statistical analysis of diffusion tensor data. Signal Process. 87(2), 250-262 (2007)

16. Florack, L., van Assen, H.: Multiplicative calculus in biomedical image analysis. J. Math. Imaging Vis. 42(1), 64-75 (2012)

17. Florack, L.M.J.: Image Structure, Computational Imaging and Vision Series, vol. 10. Kluwer Academic Publishers, Dordrecht, The Netherlands (1997)

18. Florack, L.M.J., Astola, L.J.: A multi-resolution framework for diffusion tensor images. In: Aja Fernández, S., de Luis Garcia, R. (eds.) CVPR Workshop on Tensors in Image Processing and Computer Vision, Anchorage, Alaska, USA, IEEE (2008). Digital proceedings

19. Florack, L.M.J., Haar Romeny, B.M.t., Koenderink, J.J., Viergever, M.A.: Linear scale-space. J. Math. Imaging Vis. 4(4), 325-351 (1994)

20. Fuster, A., Astola, L.J., Florack, L.M.J.: A Riemannian scalar measure for diffusion tensor images. In: Jiang, X., Petkov, N. (eds.) Proceedings of the 13th International Conference on Computer Analysis of Images and Patterns, CAIP 2009 (September 2-4 2009, Münster, Germany), Lecture Notes in Computer Science, vol. 5702, pp. 419-426. Springer, Berlin (2009)

21. Fuster, A., Dela Haije, T., Tristán-Vega, A., Plantinga, B., Westin, C.F., Florack, L.: Adjugate diffusion tensors for geodesic tractography in white matter. J. Math. Imaging Vis. 54(1), 1-14 (2016)

22. Fuster, A., Tristan-Vega, A., Dela Haije, T., Westin, C.F., Florack, L.: A novel Riemannian metric for geodesic tractography in DTI. In: O’Donnell, L., Schultz, T., Nedjati-Gilani, G., Panagiotaki, E. (eds.) MICCAI Workshop on Computational Diffusion MRI, pp. 47-54. Japan, Nagoya (2013)

23. Hagmann, P., Jonasson, L., Maeder, P., Thiran, J.P., Wedeen, V.J., Meuli, R.: Understanding diffusion MR imaging techniques: From scalar diffusion-weighted imaging to diffusion tensor imaging and beyond. RadioGraphics 26, S205-S223 (2006)

24. Hao, X., Whitaker, R.T., Fletcher, P.T.: Adaptive Riemannian metrics for improved geodesic tracking of white matter. In: Székely, G., Hahn, H.K. (eds.) Proceedings of the Twenty-Second International Conference on Information Processing in Medical Imaging-IPMI 2011 (Kloster Irsee, Germany), Lecture Notes in Computer Science, vol. 6801, pp. 13-24. Springer, Berlin (2011)

25. Hao, X., Zygmunt, K., Whitaker, R.T., Fletcher, P.T.: Improved segmentation of white matter tracts with adaptive Riemannian metrics. Med. Image Anal. 18, 161-175 (2014)

26. Hauberg, S., Schober, M., Liptrot, M., Hennig, P., Feragen, A.: A random Riemannian metric for probabilistic shortest-path tractography. In: Navab, N., Hornegger, J., Wells, W.M., Frangi, A. (eds.) Proceedings of the 18th International Conference on Medical Image Computing and Computer Assisted Intervention-MICCAI 2015 (Munich, Germany, October 5-9 2015), Lecture Notes in Computer Science, vol. 9349, pp. 597-604. Springer, Berlin (2015)

27. Higham, N.J.: Functions of Matrices: Theory and Computation. SIAM (2008) 
28. Jackowski, M., Kao, C.Y., Qiu, M., Costable, R.T., Staib, L.H.: White matter tractography by anisotropic wavefront evolution and diffusion tensor imaging. Med. Image Anal. 9, 427-440 (2005)

29. Koenderink, J.J.: The structure of images. Biol. Cybern. 50, 363-370 (1984)

30. Le Bihan, D., Mangin, J.F., Poupon, C., Clark, C.A., Pappata, S., Molko, N., Chabriat, H.: Diffusion tensor imaging: concepts and applications. J. Magn. Reson. Imaging 13, 534-546 (2001)

31. Lenglet, C., Deriche, R., Faugeras, O.: Inferring white matter geometry from diffusion tensor MRI: Application to connectivity mapping. In: Pajdla, T., Matas, J. (eds.) Proceedings of the Eighth European Conference on Computer Vision (Prague, Czech Republic, May 2004), Lecture Notes in Computer Science, vol. 3021-3024, pp. 127-140. Springer, Berlin (2004)

32. Lenglet, C., Prados, E., Pons, J.P.: Brain connectivity mapping using Riemannian geometry, control theory and PDEs. SIAM J. Imaging Sci. 2(2), 285-322 (2009)

33. Lenglet, C., Rousson, M., Deriche, R., Faugeras, O.: Statistics on the manifold of multivariate normal distributions: Theory and application to diffusion tensor MRI processing. J. Math. Imaging Vis. 25(3), 423-444 (2006)

34. Lindeberg, T.: Scale-Space Theory in Computer Vision. The Kluwer International Series in Engineering and Computer Science. Kluwer Academic Publishers, Dordrecht, The Netherlands (1994)

35. Maier-Hein, K., Neher, P., Houde, J.C., Caruyer, E., Daducci, A., Dyrby, T., Stieltjes, B., Descoteaux, M.: Tractography Challenge ISMRM 2015 High-resolution Data (2017). https:// doi.org/10.5281/zenodo.579933,

36. Maier-Hein, K.H., Neher, P., Houde, J., Côté, M., Garyfallidis, E., Zhong, J., Chamberland, M., et al.: Tractography-based connectomes are dominated by false-positive connections. bioRxiv (2016). https://doi.org/10.1101/084137, https://www.biorxiv.org/content/early/2016/ $11 / 21 / 084137$

37. Maier-Hein, K.H., Neher, P., Houde, J., Côté, M., Garyfallidis, E., Zhong, J., Chamberland, M., et al.: The challenge of mapping the human connectome based on diffusion tractography. Nat. Commun. 8(1), 1349 (2017)

38. Mori, S.: Introduction to Diffusion Tensor Imaging. Elsevier, Amsterdam (2007)

39. Neher, P.F., Descoteaux, M., Houde, J.C., Stieltjes, B., Maier-Hein, K.H.: Strengths and weaknesses of state of the art fiber tractography pipelines - a comprehensive in-vivo and phantom evaluation study using Tractometer. Med. Image Anal. 26(1), 287-305 (2015)

40. Neher, P.F., Laun, F.B., Stieltjes, B., Maier-Hein, K.H.: Fiberfox: Facilitating the creation of realistic white matter software phantoms. Magn. Reson. Med. 72, 1460-1470 (2014)

41. Novikov, D.S., Fieremans, E., Jensen, J.H., Helpern, J.A.: Random walks with barriers. Nature 7, 508-514 (2011)

42. Novikov, D.S., Kiselev, V.: Effective medium theory of a diffusion-weighted signal. NMR Biomed. 23, 682-697 (2010)

43. O'Donnell, L., Haker, S., Westin, C.F.: New approaches to estimation of white matter connectivity in diffusion tensor MRI: Elliptic PDEs and geodesics in a tensor-warped space. In: Proceedings of Medical Imaging, Computing and Computer Assisted Intervention, Lecture Notes in Computer Science, vol. 2488, pp. 459-466. Springer, Berlin (2002)

44. Özarslan, E., Koay, C.G., Shepherd, T.M., Komlosh, M.E., İrfanoğlu, M.O., Pierpaoli, C., Basser, P.J.: Mean apparent propagator (MAP) MRI: a novel diffusion imaging method for mapping tissue microstructure. Neuroimage 78, 16-32 (2013)

45. Parker, G.J.M., Wheeler-Kingshott, C.A.M., Barker, G.J.: Estimating distributed anatomical connectivity using fast marching methods and diffusion tensor imaging. IEEE Trans. Med. Imaging 21(5), 505-512 (2002)

46. Pennec, X., Fillard, P., Ayache, N.: A Riemannian framework for tensor computing. Int. J. Comput. Vis. 66(1), 41-66 (2006)

47. Pierpaoli, C., Jezzard, P., Basser, P.J., Barnett, A., Di Chiro, G.: Diffusion tensor MR imaging of the human brain. Radiology 201(3), 637-648 (1996) 
48. Piuze, E., Sporring, J., Siddiqi, K.: Maurer-Cartan forms for fields on surfaces: Application to heart fiber geometry. IEEE Trans. Pattern Anal. Mach. Intell. 37(12), 2492-2504 (2015)

49. Poupon, C., Rieul, B., Kezele, I., Perrin, M., Poupon, F., Mangin, J.F.: New diffusion phantoms dedicated to the study and validation of HARDI models. Magn. Reson. Med. 60, 1276-1283 (2008)

50. Prados, E., Soatto, S., Lenglet, C., Pons, J.P., Wotawa, N., Deriche, R., Faugeras, O.: Control theory and fast marching techniques for brain connectivity mapping. In: Proceedings of the IEEE Computer Society Conference on Computer Vision and Pattern Recognition (New York, USA, June 2006), vol. 1, pp. 1076-1083. IEEE Computer Society Press (2006)

51. Pujol, S., et al.: The DTI Challenge: towards standardized evaluation of diffusion tensor imaging tractography for neurosurgery. J. Neuroimaging 25(6), 875-882 (2015)

52. Rund, H.: The Hamilton-Jacobi Theory in the Calculus of Variations. Robert E. Krieger Publishing Company, Huntington, N.Y. (1973)

53. Rutten, G.J.M., Kristo, G., Pigmans, W., Peluso, J., Verheul, H.B.: Het gebruik van MRtractografie in de dagelijkse neurochirurgische praktijk. Tijdschrift voor Neurologie Neurochirurgie 115(4), 204-211 (2014). With English abstract

54. Savadjiev, P., Strijkers, G.J., Bakermans, A.J., Piuze, E., Zucker, S.W., Siddiqi, K.: Heart wall myofibers are arranged in minimal surfaces to optimize organ function. Proc. Natl. Acad. Sci. 109(24), 9248-9253 (2012)

55. Schober, M., Kasenburg, N., Feragen, A., Hennig, P., Hauberg, S.: Probabilistic shortest path tractography in DTI using Gaussian process ODE solvers. In: Golland, P., Hata, N., Barillot, C., Hornegger, J., Howe, R. (eds.) Proceedings of the 17th International Conference on Medical Image Computing and Computer Assisted Intervention-MICCAI 2014 (Boston, US, September 14-18 2014), Lecture Notes in Computer Science, vol. 8675, pp. 265-272. Springer, Berlin (2014)

56. Sebastiani, G., De Pasquale, F., Barone, P.: Quantifying human brain connectivity from diffusion tensor MRI. J. Math. Imaging Vis. 25(2), 227-244 (2006)

57. Sen, P.N., Basser, P.J.: Modeling diffusion in white matter in the brain: a composite porous medium. J. Magn. Reson. Imaging 23(2), 215-220 (2005)

Open Access This chapter is licensed under the terms of the Creative Commons Attribution 4.0 International License (http://creativecommons.org/licenses/by/4.0/), which permits use, sharing, adaptation, distribution and reproduction in any medium or format, as long as you give appropriate credit to the original author(s) and the source, provide a link to the Creative Commons licence and indicate if changes were made.

The images or other third party material in this chapter are included in the chapter's Creative Commons licence, unless indicated otherwise in a credit line to the material. If material is not included in the chapter's Creative Commons licence and your intended use is not permitted by statutory regulation or exceeds the permitted use, you will need to obtain permission directly from the copyright holder.

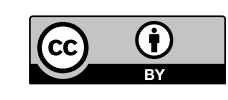

